\title{
A statistical analysis of the location and width of Saturn's southern auroras
}

\author{
S. V. Badman ${ }^{1}$, S. W. H. Cowley ${ }^{1}$, J.-C. Gérard ${ }^{2}$, and D. Grodent ${ }^{2}$ \\ ${ }^{1}$ Department of Physics \& Astronomy, University of Leicester, Leicester LE1 7RH, UK \\ ${ }^{2}$ LPAP, Université de Liège, Allée du 6 Août - Sart Tilman, B4000 Liège, Belgium
}

Received: 31 August 2006 - Revised: 23 November 2006 - Accepted: 27 November 2006 - Published: 21 December 2006

\begin{abstract}
A selection of twenty-two Hubble Space Telescope images of Saturn's ultraviolet auroras obtained during 1997-2004 has been analysed to determine the median location and width of the auroral oval, and their variability. Limitations of coverage restrict the analysis to the southern hemisphere, and to local times from the post-midnight sector to just past dusk, via dawn and noon. It is found that the overall median location of the poleward and equatorward boundaries of the oval with respect to the southern pole are at $\sim 14^{\circ}$ and $\sim 16^{\circ}$ co-latitude, respectively, with a median latitudinal width of $\sim 2^{\circ}$. These median values vary only modestly with local time around the oval, though the poleward boundary moves closer to the pole near noon $\left(\sim 12.5^{\circ}\right)$ such that the oval is wider in that sector (median width $\sim 3.5^{\circ}$ ) than it is at both dawn and dusk $\left(\sim 1.5^{\circ}\right)$. It is also shown that the position of the auroral boundaries at Saturn are extremely variable, the poleward boundary being located between $2^{\circ}$ and $20^{\circ}$ co-latitude, and the equatorward boundary between $6^{\circ}$ and $23^{\circ}$, this variability contrasting sharply with the essentially fixed location of the main oval at Jupiter. Comparison with Voyager plasma angular velocity data mapped magnetically from the equatorial magnetosphere into the southern ionosphere indicates that the dayside aurora lie poleward of the main upward-directed field-aligned current region associated with corotation enforcement, which maps to $\sim 20^{\circ}-24^{\circ}$ co-latitude, while agreeing reasonably with the position of the open-closed field line boundary based on estimates of the open flux in Saturn's tail, located between $\sim 11^{\circ}$ and $\sim 15^{\circ}$. In this case, the variability in location can be understood in terms of changes in the open flux present in the system, the changes implied by the Saturn data then matching those observed at Earth as fractions of the total planetary flux. We infer that the broad (few degrees) diffuse auroral emissions and sub-corotating auroral patches observed in the dayside
\end{abstract}

Correspondence to: S. V. Badman

(svb4@ion.le.ac.uk) sector at Saturn result from precipitation from hot plasma sub-corotating in the outer magnetosphere in a layer a few Saturn radii wide adjacent to the magnetopause, probably having been injected either by Dungey-cycle or Vasyliunascycle dynamics on the nightside.

Keywords. Magnetospheric physics (Auroral phenomena; Magnetosphere-ionosphere interactions; Planetary magnetospheres)

\section{Introduction}

The first indications that auroral emissions occur in Saturn's polar regions were obtained by the ultraviolet (UV) photometer onboard the Pioneer-11 spacecraft during its flyby in 1979 (Judge et al., 1980), and remotely from observations by the IUE spacecraft (Clarke et al., 1981; McGrath and Clarke, 1992). The first unambiguous detections were made by the two Voyager spacecraft in 1980 and 1981, revealing emissions poleward of $\sim 12^{\circ}$ co-latitude in both hemispheres that were extended in longitude, but with some localised brightenings (Broadfoot et al., 1981; Sandel and Broadfoot, 1981; Sandel et al., 1982). Subsequent observations have been made of both the northern and southern hemisphere auroras using the Faint Object Camera, Wide Field Planetary Camera 2, and Space Telescope Imaging Spectrograph (STIS) instruments on the Hubble Space Telescope (HST) (e.g. Gérard et al., 1995, 2004; Trauger et al., 1998; Cowley et al., 2004a; Prangé et al., 2004; Grodent et al., 2005). These have revealed some common features, mainly thin circumpolar auroral arcs and diffuse auroral patches, lying at co-latitudes between $\sim 10^{\circ}$ and $\sim 20^{\circ}$, and often enhanced at dawn relative to dusk. However, the HST images have also revealed considerable variability in the location of the observed features, and in the intensity of the emissions, which ranges

Published by Copernicus GmbH on behalf of the European Geosciences Union. 
from below an instrumental threshold of a few $\mathrm{kR}$ to over $120 \mathrm{kR}$.

An auroral oval can be generated either as a result of the interaction between a planet's magnetosphere and the solar wind, or internally due to processes associated with the enforcement of planetary rotation on magnetospheric plasma. Auroras associated with the solar wind interaction are located at and near the boundary between open and closed field lines surrounding the magnetic pole, such that their latitudinal position varies with the amount of open flux in the system, as observed at Earth (e.g. Milan et al., 2003, 2004, 2006). These auroras consist of "discrete" forms associated with the upward-directed field-aligned currents generated by magnetosphere-ionosphere momentum exchange, carried by precipitating accelerated magnetospheric electrons, together with "diffuse" auroras produced by charged particle precipitation from regions of hot magnetospheric plasma. Hot plasmas are generated, for example, in regions downstream from reconnection sites associated with the cyclical magnetospheric flow driven by the solar wind, termed here the Dungey-cycle (Dungey, 1961). These auroral components are strongly modulated by prevailing solar wind conditions, and will generally also present persistent asymmetries in local time (e.g. Jackman and Cowley, 2006).

Auroras associated with planetary rotation are firstly related to the field-aligned current system generated by angular momentum transfer between the planetary atmosphere and the magnetospheric plasma (Hill, 1979). For the magnetic field polarity at Jupiter and Saturn, these currents are directed upward in the inner magnetospheric region where near-rigid corotation breaks down, and may produce "discrete" auroras on these field lines if the currents are sufficiently strong (e.g. Cowley and Bunce, 2001; Hill, 2001). If these currents flow sufficiently deeply inside the near-axisymmetric magnetosphere then local time effects are expected to be weak, and they will also be relatively steady over time if internal plasma sources and transport are relatively steady. These properties then describe the principal features exhibited by the jovian main auroral oval, though these auroras do vary strongly with planetary longitude due to asymmetries in the internal magnetic field of the planet (e.g. Grodent et al., 2003). Transient modulation of these auroras may result from magnetospheric compressions and expansions due to rapid changes in solar wind dynamic pressure, if these are sufficient to alter the magnetospheric plasma angular velocity profile in the relevant region (Southwood and Kivelson, 2001; Cowley and Bunce, 2003a, b; Gong and Hill, 2005; Nichols et al., 2006). Auroras associated with internal processes may also occur at higher latitudes due to the Vasyliunas-cycle of internal plasma mass loss via down-tail plasmoid ejection (Vasyliunas, 1983). These auroras will occur in the region directly equatorward of those associated with the Dungey-cycle, and will also generally exhibit local time asymmetries imposed by the flow of the solar wind (Cowley et al., 2004a). The principal distinction from the tail reconnection process asso- ciated with the Dungey-cycle is that Vasyliunas-cycle reconnection does not involve any change in the amount of open flux present in the system.

In general, these solar wind and internally generated processes can occur simultaneously, potentially resulting in two auroral regions, one located in the vicinity of the open-closed field line boundary and the other at lower latitudes mapping to the corotation-breakdown region. However, Cowley and Bunce (2003c) presented an empirical model of corotation-related coupling currents in Saturn's magnetosphere, based on Voyager spacecraft velocity data, and concluded that the large-scale upward-directed currents, flowing at $\sim 20^{\circ}-25^{\circ}$ co-latitude, are too weak to generate bright auroras. However, smaller-scale flow features which are perhaps due to moon effects may produce weak $(\sim 1 \mathrm{kR})$ narrow auroral rings or partial rings at co-latitudes $\sim 20^{\circ}$. This model was subsequently extended to higher latitudes by Cowley et al. (2004b), showing that the upward current at the open-closed field line boundary is expected to be of sufficient intensity to generate auroras of a few tens of $\mathrm{kR}$, mapping to $\sim 14^{\circ}$ co-latitude in the southern hemisphere. Corresponding features in the northern hemisphere are expected to be located $\sim 1.5^{\circ}$ closer to the pole due to the inter-hemispheric asymmetry in the magnetic field associated with the quadrupole internal field component (Davis and Smith, 1990; Cowley and Bunce, 2003c). On the basis of these results, Cowley et al. (2004b) concluded that Saturn's observed auroras are more likely to be associated with the open-closed field line boundary and the solar wind interaction, rather than with the corotation-enforcement current system.

The purpose of the present paper is to contribute to an understanding of Saturn's auroras by providing statistics on the position and width of the observed auroral features. This study incorporates all the available images from HST/STIS, obtained during the interval from 1997 to 2004. During this interval the tilt of Saturn's spin axis relative to Earth was such that only the southern hemisphere auroras could be observed. In the next section we first describe the images and how they were selected for inclusion in the study. Section 2.2 then describes the process of determining the location and width of the auroral oval, and the statistical methods employed. The statistics are presented and interpreted in Sect. 3, and the paper concludes with a discussion of the results in Sect. 4.

\section{Observations}

\subsection{Data description and selection}

The data set employed in this study consists of the full set of HST images of Saturn's southern auroras that were obtained between 11 October 1997 and 30 January 2004, during the active lifetime of the STIS instrument. The images were obtained using the FUV photon-counting detector 
MAMA (Multi-Anode Microchannel Array) in either unfiltered ("clear") mode or filtered mode. In the clear mode the detector has a bandpass between 115 and $180 \mathrm{~nm}$, while the filtered mode uses a $\mathrm{SrF}_{2}$ filter which rejects wavelengths below $\sim 125 \mathrm{~nm}$, including the strong H Lyman- $\alpha$ emission line and potential geocoronal contamination. The MAMA array has a pixel size of $0.0243 \mathrm{arcsec}$ and a point spread function (PSF) of 0.08 arcsec full width at half maximum.

From the total of 83 images obtained over the above interval, often 2 or 4 images were obtained consecutively on the same HST orbit, revealing essentially the same auroral features. To include all of these images without selection would thus bias the statistical results. Observations were also occasionally made on consecutive orbits spaced by $\sim 90 \mathrm{~min}$, also displaying similar features. More recent observations presented by Gérard et al. (2006) obtained using the HST Advanced Camera for Surveys instrument and not included in this study, have shown that images separated by $\sim 5 \mathrm{~h}$ (or more) generally reveal significantly different morphologies. Therefore in this study we use only those images spaced by $5 \mathrm{~h}$, i.e. by approximately half a Saturn rotation, or more. Comparison of consecutive filtered and clear images from January 2004 has also shown that the auroral features present in both are not noticeably displaced with respect to each other. It therefore seems reasonable to include both filtered and clear images in our analysis of the aurora's location, though to maintain consistency, if both filtered and clear images are available, the clear image has been used preferentially. Where two clear images were obtained consecutively on the same orbit, i.e. in the January 2004 set, the two images have been summed to increase the signal to noise ratio. In the cases where two clear images were obtained on consecutive orbits spaced by $\sim 90 \mathrm{~min}$, the first of the two images has been used in this study. Details of the 22 images and summed images selected for use in this study according to the above criteria are listed in Table 1.

For each image, the STIS field of view was projected onto a planetary latitude-longitude grid using a limb-fitting method described by Grodent et al. (2003, 2005). A selection of six projected images is shown in Fig. 1, revealing the increasingly complete view of the southern auroral region accessible from 1997 to 2004, as well as the variable morphology and intensity of the observed aurora. The geometry of these plots is such that the observer is looking "through" the planet from the northern into the southern ionosphere, with the sunward direction at the bottom of each image, dawn to the left, and dusk to the right. Longitude in these figures is defined anti-clockwise from midnight, such that dawn corresponds to $90^{\circ}$, noon to $180^{\circ}$, and dusk to $270^{\circ}$. Note that the term "longitude" used throughout this study is thus equivalent to local time, and is not a coordinate fixed to the rotating planet. Wherever local times (LT) are also quoted they are written in the format h:min. The region from the pole to $30^{\circ}$ co-latitude is shown in the figure, marked at intervals of $10^{\circ}$ co-latitude. Auroral intensities in kR are colour-coded according to the scale shown on the right hand side of the figure.

Panels (a) to (f) in Fig. 1 correspond to images 1, 5, 6, 18, 20 and 21 in Table 1. The aurora in Fig. 1a, observed on 11 October 1997, is of a broad and diffuse nature, covering a longitude range from $\sim 80^{\circ}$ to $\sim 150^{\circ}(05: 20-10: 00 \mathrm{LT})$ in the morning sector, centred on $\sim 16^{\circ}$ to $\sim 17^{\circ}$ co-latitude. The auroras observed on 7 December 2000, shown in Fig. 1b, are again broad and centred on $\sim 17^{\circ}$ co-latitude, but are more intense and extend in a spiral form from $\sim 30^{\circ}$ to $\sim 280^{\circ}$ longitude ( $\sim 02: 00-18: 40 \mathrm{LT})$, narrowing to $\sim 2^{\circ}-3^{\circ}$ width at dawn and noon and becoming dim post-noon. In contrast, Fig. 1c, obtained on 8 December 2000, reveals a very narrow $\left(\sim 1^{\circ}\right.$ co-latitude) auroral arc extending from pre-dawn to post-noon (from $\sim 40^{\circ}$ to $\sim 200^{\circ}$ longitude) centred on $\sim 18^{\circ}$ co-latitude, with negligible emission on the dusk side. Figure 1d shows two distinct regions of aurora observed on 23 January 2004. In this example there is a narrow band of bright emission at $\sim 15^{\circ}$ co-latitude in the dawn sector, but also a diffuse feature at noon that is several degrees wide in co-latitude, lying poleward of the dawn emission at $\sim 12^{\circ}$ to $\sim 15^{\circ}$ co-latitude. Such noon features have been discussed in terms of magnetopause-related dayside cusp emissions by Bunce et al. (2005) and Gérard et al. (2005). The next image shown in Fig. 1e is a uniquely-observed feature from 26 January 2004. Virtually the entire dawn side of the polar cap is filled with very bright diffuse emissions, extending from $\sim 2^{\circ}$ to $\sim 18^{\circ}$ co-latitude. There is negligible emission on the dusk side except for a narrow feature post-noon at $\sim 6^{\circ}$ co-latitude. The final image shown in Fig. 1f is of another spiral feature, observed on 28 January 2004, which is generally brighter, broader and located closer to the pole (centred at $\sim 12^{\circ}$ colatitude) than that shown in Fig. 1b. The intense emission extends from $\sim 10^{\circ}$ to $\sim 300^{\circ}$ longitude $(\sim 00: 40-20: 00 \mathrm{LT})$ and is narrower $\left(\sim 1^{\circ}-3^{\circ}\right.$ co-latitude) beyond $\sim 200^{\circ}$ longitude $(\sim 13: 20 \mathrm{LT})$. Overall, these images demonstrate the wide variability in the form and location of Saturn's auroras, to be quantified statistically in the sections below.

\subsection{Determining the auroral boundaries and widths}

The white crosses on each image in Fig. 1 mark the poleward and equatorward boundaries of the auroral emission, determined using the method now to be described. The latitudinal positions of the auroral boundaries were initially located by eye, looking for peaks in the emission intensity along a given meridian. For simple one-peak profiles the boundaries were then determined as the half-peak power points above the background emission. Two such examples are shown in Fig. 2 over the co-latitudinal range $0^{\circ}-30^{\circ}$, where the red and blue vertical dashed lines mark the poleward and equatorward boundaries, respectively, so determined. Figure 2a is an example of a narrow auroral feature at $90^{\circ}$ longitude observed on 8 December 2000 (Fig. 1c). Figure 2b shows a broader emission profile observed at $140^{\circ}$ longitude on 23 
Table 1. Properties of the 22 HST images used in this study.

\begin{tabular}{|c|c|c|c|c|c|c|}
\hline No. & $\begin{array}{c}\text { Date } \\
\mathrm{dd} / \mathrm{mm} / \mathrm{yy}\end{array}$ & Start time/UT & Exposure time/s & Filter & Sub-Earth latitude/deg & Max. brightness $/ \mathrm{kR}$ \\
\hline 1 & 11 Oct 1997 & 0415 & 774 & Clear & -10.3 & 50 \\
\hline 2 & 11 Oct 1997 & 1042 & 754 & Clear & -10.3 & 29 \\
\hline 3 & 20 Nov 1997 & 1347 & 1000 & Clear & -8.9 & 45 \\
\hline 4 & 5 Dec 1997 & 0530 & 600 & Clear & -8.7 & 28 \\
\hline 5 & 7 Dec 2000 & 1130 & 480 & $\mathrm{SrF}_{2}$ & -23.3 & 108 \\
\hline 6 & 8 Dec 2000 & 1000 & 480 & $\mathrm{SrF}_{2}$ & -23.3 & 52 \\
\hline 7 & 28 Jan 2001 & 0847 & 330 & $\mathrm{SrF}_{2}$ & -23.1 & 40 \\
\hline 8 & 29 Jan 2001 & 1029 & 300 & $\mathrm{SrF}_{2}$ & -23.1 & 56 \\
\hline 9 & 8 Jan 2004 & 0442 & 540 & Clear & -25.7 & 49 \\
\hline 10 & 8 Jan 2004 & 1104 & 540 & Clear & -25.7 & 31 \\
\hline 11 & 10 Jan 2004 & 0441 & 540 & Clear & -25.7 & 31 \\
\hline 12 & 12 Jan 2004 & 0928 & 540 & Clear & -25.7 & 47 \\
\hline 13 & 14 Jan 2004 & 0441 & 540 & Clear & -25.8 & 29 \\
\hline 14 & 16 Jan 2004 & 0128 & 540 & Clear & -25.8 & 27 \\
\hline 15 & 18 Jan 2004 & 0440 & 540 & Clear & -25.8 & 82 \\
\hline 16 & 20 Jan 2004 & 0128 & 540 & Clear & -25.8 & 43 \\
\hline 17 & 21 Jan 2004 & 2040 & 540 & Clear & -25.9 & 29 \\
\hline 18 & 23 Jan 2004 & 0439 & 540 & Clear & -25.9 & 30 \\
\hline 19 & 24 Jan 2004 & 2351 & 540 & Clear & -25.9 & 47 \\
\hline 20 & 26 Jan 2004 & 1902 & 540 & Clear & -25.9 & 83 \\
\hline 21 & 28 Jan 2004 & 0127 & 540 & Clear & -26.0 & 122 \\
\hline 22 & 30 Jan 2004 & 1901 & 540 & Clear & -26.0 & 64 \\
\hline
\end{tabular}

January 2004 (Fig. 1d). For more complicated profiles, e.g. those that have multiple peaks, the boundary was similarly determined by marking the half-power point in the sharp change in emission intensity on either side of the intensity peaks. Using this method, both the poleward and equatorward boundaries of the emission were recorded at $0.1^{\circ}$ latitudinal accuracy at every ten degrees longitude ( $40 \mathrm{~min}$ LT) in each image. The width of the emission was then obtained simply from the difference between the poleward and equatorward positions, with a correction for broadening of features by the PSF to be discussed below. Clearly, the boundaries can only be marked where there is a discernable peak in emission above the background level, such that both poleward and equatorward boundaries can be determined. The number of such "good" data points at each longitude is plotted in Fig. 3, where longitude is defined as above. The axis at the top of the plot shows the equivalent local time for reference. The distribution peaks broadly at values of 17-19, out of a possible maximum of 22 , between $90^{\circ}$ (dawn) and $180^{\circ}$ (noon), before falling gradually in the post-noon hours to 9 at $270^{\circ}$ longitude (dusk). The number of "good" points falls to low numbers $(<7)$ for the longitude range $290^{\circ}-40^{\circ}$ (via midnight). The asymmetry about noon results from the fact that bright auroral features are more commonly observed on the dawn side and at noon than in the afternoon sector. Observations on the nightside are limited in the 1997 data set (see Fig. 1a), and features visible around midnight become stretched by the projection method, such that there are few "good" data points in the midnight sector. In Fig. 3 the vertical dashed lines at $50^{\circ}$ and $280^{\circ}$ longitude mark the limits of the range of longitudes over which we have chosen to perform the statistical analysis, based on there being at least a minimum number of 9 "good" data points. This range extends from the post-midnight sector to just past dusk, via dawn and noon (03:20-18:40 LT).

Before discussing the distributions of auroral boundary positions and widths the limitations imposed by broadening of the auroral features by the instrument PSF of 0.08 arcsec full width at half maximum must be considered. At the distance of Saturn, this corresponds to a distance transverse to the line-of-sight of $\sim 500 \mathrm{~km}$. At the sub-Earth point on the planet this is equivalent to a latitudinal spread of $\Delta \theta_{\mathrm{PSF}} \sim 0.5^{\circ}$. Away from the sub-Earth point the equivalent latitude range increases due to the inclination of the planetary surface to the line-of-sight, but this increase is negligible near the ansae of the oval at dawn and dusk. On the noon meridian, however, the projected latitude range corresponding to the PSF increases to $\Delta \theta_{\mathrm{PSF}}^{\prime} \approx \Delta \theta_{\mathrm{PSF}} / \cos \lambda$, where $\lambda$ is the auroral latitude relative to the sub-Earth point, which is typically $\sim-50^{\circ}$ for the data examined here (i.e. the auroral latitude relative to the planet's equator is $\sim-75^{\circ}$, as will be shown below, while the sub-Earth latitude 
(a) $1997-10-1104: 15: 43$

00

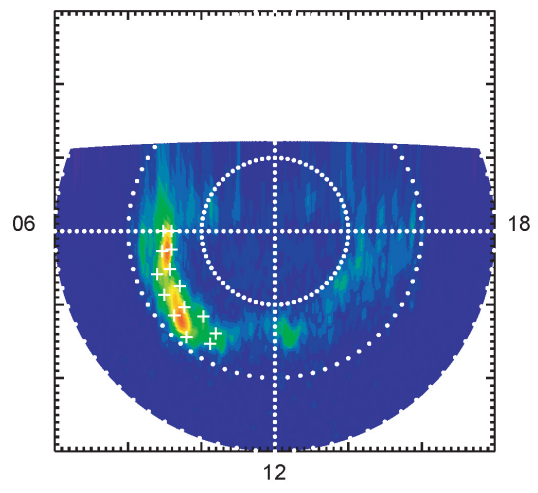

(d) $2004-01-23$ 04:39:21

00

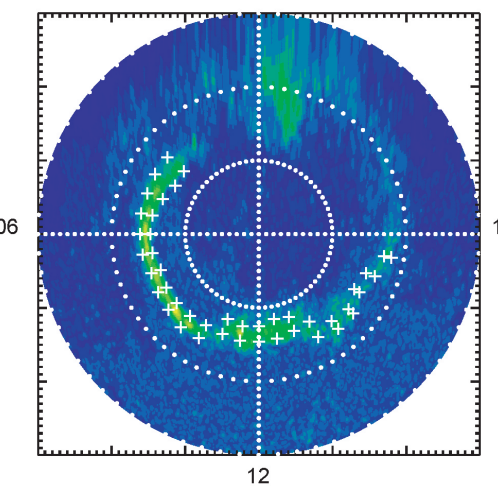

(b) 2000-12-07 11:30:03

00

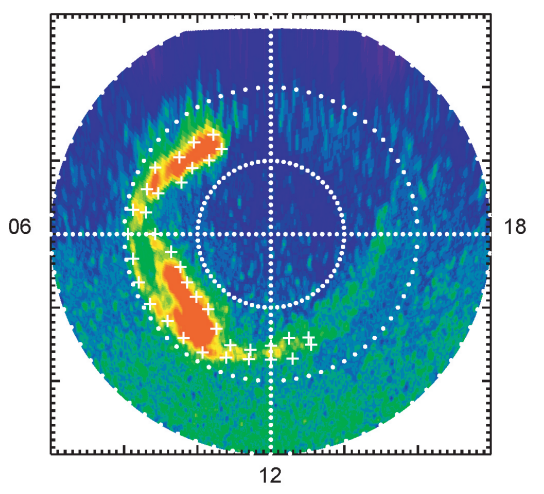

(e) 2004-01-26 19:02:32

00

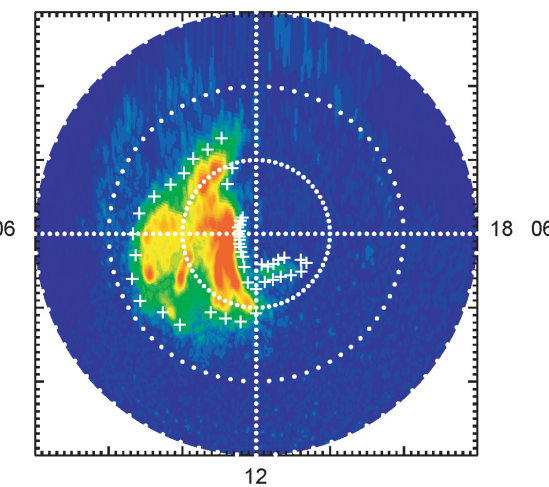

(c) 2000-12-08 10:00:27

00

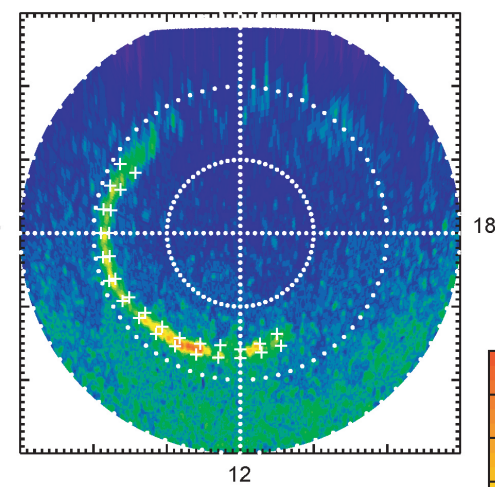

(f) $2004-01-28$ 01:27:28

00

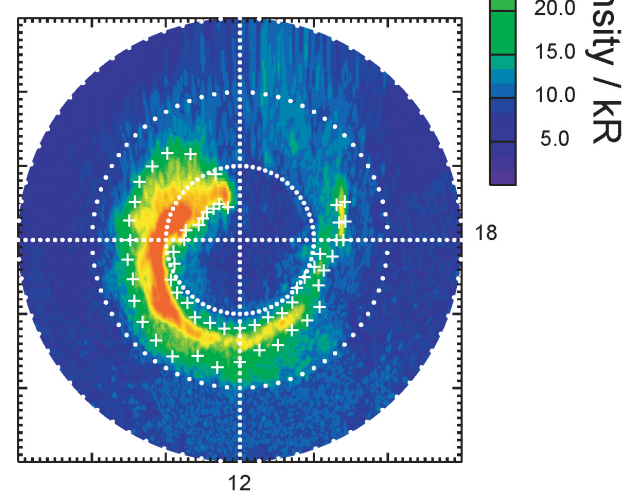

Fig. 1. Selection of six UV images of Saturn's southern aurora obtained during the interval 11 October 1997 to 30 January 2004 , with the date and start time of each image shown at the top of each plot. Panels (a) to (f) correspond to images numbered 1, 5, 6, 18, 20, and 21 in Table 1. The images are projected onto a polar grid from the pole to $30^{\circ}$ co-latitude, viewed as though looking "through" the planet onto the southern pole. Noon is at the bottom of each plot, and dawn to the left, as indicated. The UV auroral intensity is plotted according to the colour scale shown on the right-hand side of the figure. The white crosses mark the poleward and equatorward boundaries of the auroral emissions determined as discussed in the text.

is $\sim-25^{\circ}$ as in Table 1 ). At noon we thus typically have $\Delta \theta_{\mathrm{PSF}}^{\prime} \approx \Delta \theta_{\mathrm{PSF}} / \cos 50^{\circ} \approx 0.78^{\circ}$. The latitudinal spread associated with the PSF therefore increases from $\sim 0.5^{\circ}$ at dawn and dusk to $\sim 0.78^{\circ}$ near noon.

Two consequences follow from this. The first is that when forming histograms of the latitudinal distribution of the auroral boundary positions we should use a bin size that is comparable with or larger than the spread associated with the PSF. Therefore, we have chosen to use a bin size of $1^{\circ}$ latitude, a value which is also reasonable considering the positional uncertainties associated with the projection method (Grodent et al., 2005). The second consequence is that the observed auroral widths, determined as described above, will be somewhat increased compared with the actual values. It can readily be shown that if the auroral distribution is approximated as a Gaussian whose true latitudinal width is $\Delta \theta$ (full width at half maximum), then if this is convolved with a PSF which is also taken to be a Gaussian of width $\Delta \theta_{\mathrm{PSF}}^{\prime}$ (full width at half maximum), then the resulting distribution will be another Gaussian whose observed full width at half maximum will be $\Delta \theta^{\prime}=\sqrt{\Delta \theta^{2}+\Delta \theta^{\prime 2} \mathrm{PSF}}$. Consequently we can make a simple correction for the effect of the PSF on the latitudinal widths by calculating $\Delta \theta=\sqrt{\Delta \theta^{\prime 2}-\Delta \theta^{\prime} \mathrm{PSF}^{2}}$. As indicated above, $\Delta \theta_{\mathrm{PSF}}^{\prime}$ varies modestly around the portion of the auroral oval considered in this study from $\sim 0.5^{\circ}$ near dawn and dusk to $\sim 0.78^{\circ}$ near noon (for the majority of the observation intervals). Since these represent small corrections for the great majority of the data, we have chosen simply to use a fixed mean value of $\Delta \theta_{\mathrm{PSF}}^{\prime} \sim 0.64^{\circ}$. We emphasise that the correction to $\Delta \theta^{\prime}$ is small unless it is very close to $\Delta \theta_{\mathrm{PSF}}^{\prime}$. Consider for example the emission profiles shown in Fig. 2. The first (Fig. 2a) is a relatively narrow feature with $\Delta \theta^{\prime}=1.1^{\circ}$. Using the above algorithm the "corrected" width is reduced to $\Delta \theta \sim 0.9^{\circ}$, which represents only 
(a)

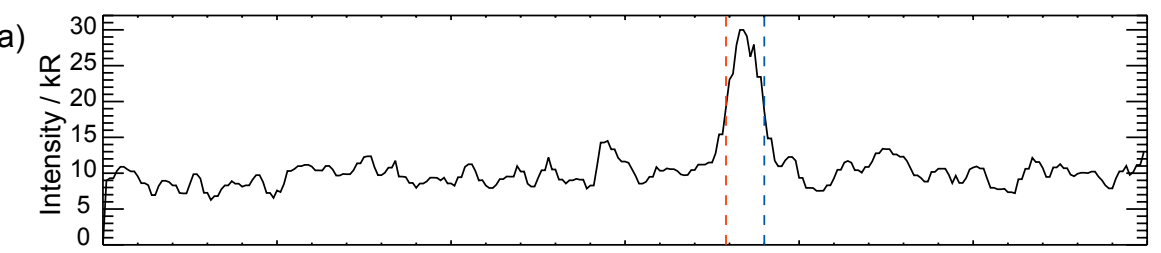

(b)

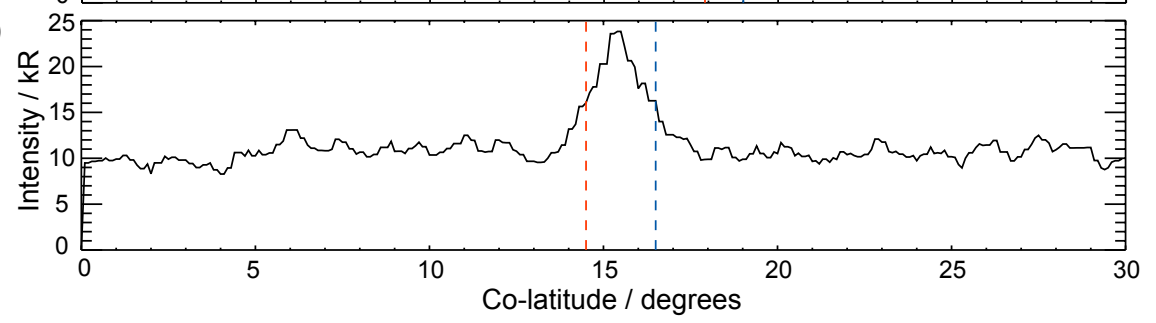

Fig. 2. Two auroral emission intensity profiles $(\mathrm{kR})$ for co-latitudes $0^{\circ}-30^{\circ}$, where the red and blue vertical dashed lines mark the poleward and equatorward boundaries, respectively, of the auroral features, determined as discussed in the text. Panel (a) shows a narrow feature observed at $90^{\circ}$ longitude (i.e. dawn) on 8 December 2000 (see Fig. 1c). Panel (b) shows a more typical broader feature observed at $140^{\circ}$ longitude (i.e. pre-noon) on 23 January 2004 (see Fig. 1d).

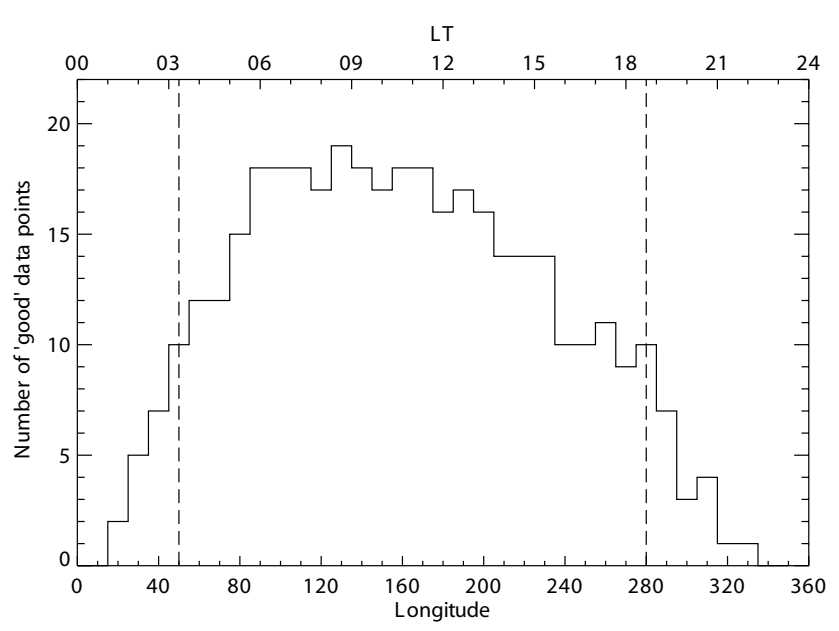

Fig. 3. Longitude distribution of "good" auroral boundary data points, i.e. those where there is a discernable peak above the background emission such that both the poleward and equatorward positions can be determined, out of the possible maximum of 22 . The vertical dashed lines mark the limits of the range of longitudes (i.e. local times) that are used in this study, based on there being at least a minimum number of 9 data points.

a modest change of $\sim 20 \%$. The second example in Fig. $2 \mathrm{~b}$ is a more typical profile with $\Delta \theta^{\prime}=2.0^{\circ}$. In this case the "corrected" value is $\Delta \theta \sim 1.9^{\circ}$, representing a difference of only $\sim 5 \%$.

\section{Distributions of auroral boundary positions and widths}

Figure 4 shows the co-latitudinal distributions of the poleward (red) and equatorward (blue) auroral boundaries for the longitude range $50^{\circ}-280^{\circ}(03: 20-18: 40 \mathrm{LT})$ in the form of histograms, where the "probability" plotted on the ordinate is the occurrence in each co-latitude bin as a fraction of the total number of "good" data points at that longitude. The red and blue vertical dashed lines mark the median co-latitudinal positions of the poleward and equatorward auroral boundaries, respectively. Considering first the poleward boundary position, the median values of the latitudinal distributions remain relatively constant over longitude, at $14^{\circ}-15^{\circ}$ co-latitude for most of the dawn sector, moving poleward to $12^{\circ}-13^{\circ}$ around noon $\left(160^{\circ}-230^{\circ}\right.$ longitude), then gradually shifting equatorward to $15^{\circ}-16^{\circ}$ at dusk. We also see, however, that the poleward boundary position is very variable, covering a large range of $2^{\circ}-20^{\circ}$ co-latitude when all longitudes are considered. The distribution is widest on the dawn side of the oval, covering this full range of $2^{\circ}-20^{\circ}$ co-latitude for virtually all dawn sector longitudes $\left(50^{\circ}-130^{\circ}\right)$. The distribution narrows to $3^{\circ}-19^{\circ}$ at $140^{\circ}$ longitude (09:20 LT) and has a local minimum of $7^{\circ}-16^{\circ}$ at noon, i.e. the location of the poleward boundary is twice as variable at dawn than at noon. This variation is also quantified by the standard deviation of the values, which decreases from a maximum of $5.5^{\circ}$ at $70^{\circ}$ longitude (04:40 LT) to $2.6^{\circ}$ at noon. Post-noon the distribution of poleward boundary positions gradually broadens again to $4^{\circ}-19^{\circ}$ co-latitude (standard deviation $3.7^{\circ}$ ) at $220^{\circ}$ longitude (14:40 LT), then narrows again to a minimum of $12^{\circ}-$ $18^{\circ}$ (standard deviation $1.6^{\circ}$ ) at $270^{\circ}$ longitude (dusk). The boundary positions with smallest co-latitude, i.e. the points at $2^{\circ}$ at dawn and $4^{\circ}$ post-noon, correspond to those observed on 26 January 2004 when the dawn side of the polar cap was 

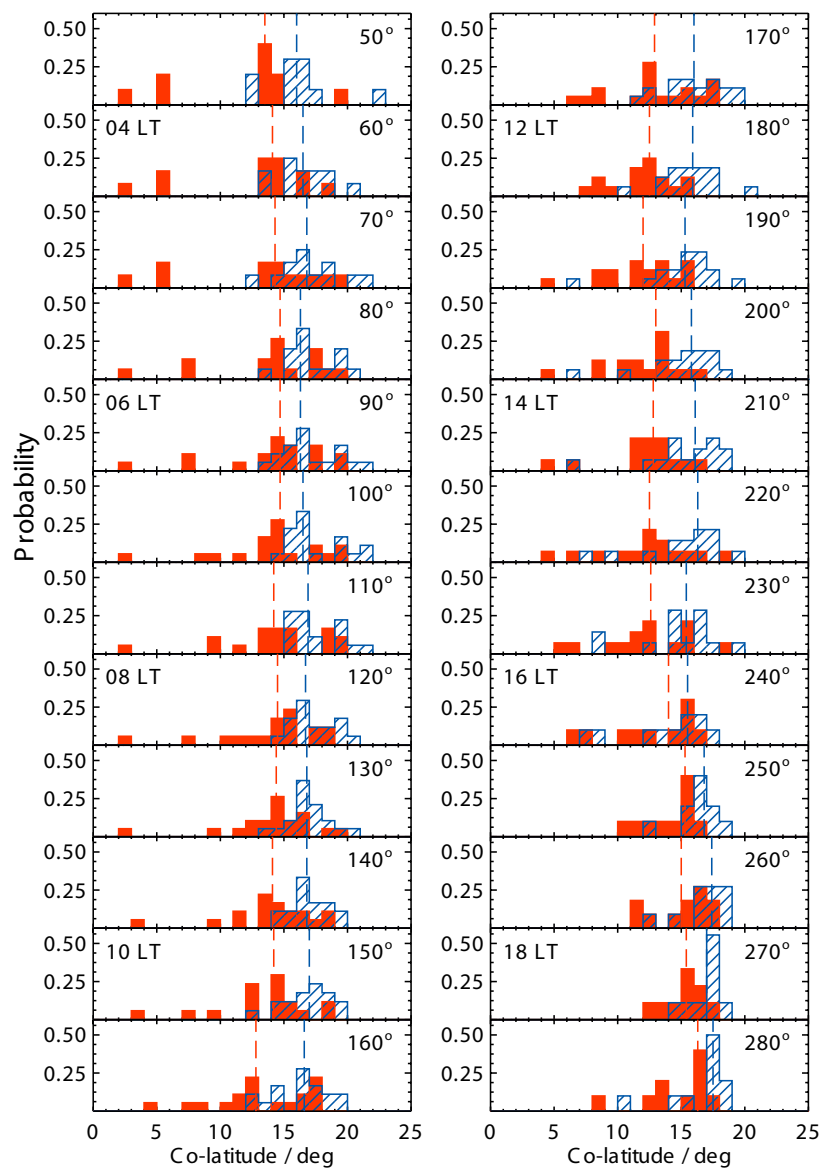

Fig. 4. Histogram showing the distribution of poleward (red) and equatorward (blue) auroral boundaries over the co-latitude range $0^{\circ}-25^{\circ}$. Data is shown for the longitude range $50^{\circ}-280^{\circ}$ in $10^{\circ}$ steps, where $0^{\circ}$ is midnight, $90^{\circ}$ dawn, $180^{\circ}$ noon, and $270^{\circ}$ dusk. The probability plotted on the ordinate is the occurrence in each colatitude bin as a fraction of the total number of "good" data points at that longitude. The vertical dashed red and blue lines mark the median co-latitude position for the poleward and equatorward boundaries, respectively, at each longitude.

virtually filled with bright emission (Fig. 1e). The most expanded poleward boundary locations $\left(20^{\circ}\right.$ in the dawn sector and $19^{\circ}$ post-noon) occur in the presence of diffuse auroral blobs and narrow arcs in the dawn sector and post-noon (similar to the features shown in Figs. 1a, c and d).

Now considering the equatorward boundary, we first see that the median values are again relatively constant over longitude. On the dawn side $\left(50^{\circ}-160^{\circ}\right.$ longitude) the median equatorward boundary position is steady at $\sim 16^{\circ}-17^{\circ}$. Around noon $\left(170^{\circ}-200^{\circ}\right.$ longitude) the median position moves slightly poleward to $15^{\circ}-16^{\circ}$ co-latitude, then varies between $15^{\circ}-18^{\circ}$ co-latitude in the remaining range of longitudes towards dusk. Turning now to the latitudinal distributions it can be seen that the boundary location is again very variable, with an overall variation in position of $6^{\circ}-23^{\circ}$

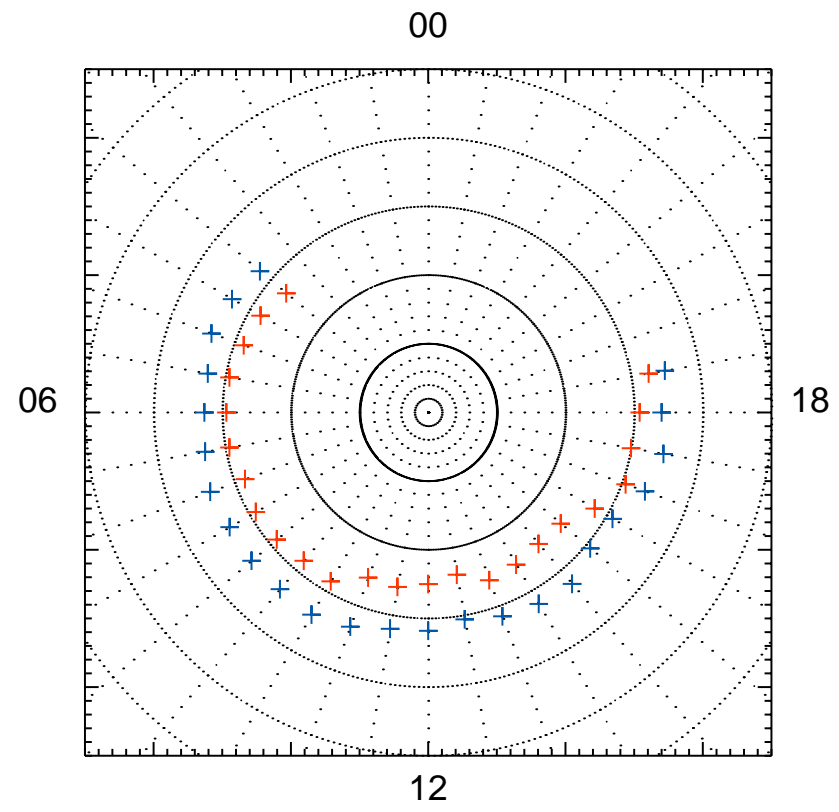

Fig. 5. The median poleward (red) and equatorward (blue) auroral boundary positions, plotted on a polar grid with the sunward direction at the bottom of the figure, dawn to the left, and dusk to the right, as in Fig. 1. Rings of co-latitude are marked at $5^{\circ}$ intervals, and lines of longitude at $10^{\circ}$ intervals.

co-latitude, only $1^{\circ}$ less than that of the poleward boundary position. However, the standard deviations of the distributions of the equatorward boundary positions are consistently smaller than those of the poleward boundary positions at each longitude implying that the equatorward boundary position is less variable. The smallest positional values that lead to such a large range are due to the presence of two high-latitude features at longitudes $190^{\circ}-240^{\circ}$ in the postnoon sector. The first was observed on 29 January 2001 as part of a bright spiral extending through dusk toward midnight, while the second was observed on 26 January 2004 as a narrow band continuing from the bright dawn emission (Fig. 1e). Pre-dawn, the equatorward boundary covers a narrower range between $12^{\circ}$ and $23^{\circ}$ co-latitude, with a typical standard deviation of $\sim 2.5^{\circ}$. Post-dawn in the morning sector, the distribution narrows somewhat, reaching a minimum range in this sector of $14^{\circ}-20^{\circ}$ (standard deviation $1.6^{\circ}$ ) at $140^{\circ}$ longitude (09:20 LT). At noon the distribution of positions is wider, $10^{\circ}-21^{\circ}$ co-latitude (standard deviation $2.2^{\circ}$ ), while the maximum range of $6^{\circ}-20^{\circ}$ is observed at $190^{\circ}$ longitude (12:40 LT), due to the presence of the high-latitude feature mentioned above. The distribution narrows duskward of this, with a minimum range of $14^{\circ}-19^{\circ}$ (standard deviation $1.3^{\circ}$ ) at $270^{\circ}$ longitude (dusk). The maximum equatorward boundary positions correspond to the same features as the maximum poleward boundary positions i.e. the presence of diffuse blobs and narrow arcs of emission. 
Table 2. Median value and standard deviation of the auroral boundary position and width at each longitude considered.

\begin{tabular}{|c|c|c|c|c|c|c|}
\hline \multirow{2}{*}{$\begin{array}{l}\text { Longitude/deg } \\
\text { (LT/h:min) }\end{array}$} & \multicolumn{2}{|c|}{ Poleward boundary position/deg } & \multicolumn{2}{|c|}{ Equatorward boundary position/deg } & \multicolumn{2}{|c|}{ Auroral oval width/deg } \\
\hline & Median & $\begin{array}{l}\text { Standard } \\
\text { deviation }\end{array}$ & Median & $\begin{array}{l}\text { Standard } \\
\text { deviation }\end{array}$ & Median & $\begin{array}{l}\text { Standard } \\
\text { deviation }\end{array}$ \\
\hline $50(03: 20)$ & 13.5 & 5.0 & 16.0 & 2.8 & 3.2 & 3.2 \\
\hline $60(04: 00)$ & 14.1 & 5.0 & 16.5 & 2.1 & 3.1 & 3.5 \\
\hline $70(04: 40)$ & 14.3 & 5.5 & 16.8 & 2.5 & 2.1 & 3.7 \\
\hline $80(05: 20)$ & 14.7 & 4.8 & 16.3 & 2.0 & 1.6 & 3.6 \\
\hline $90(06: 00)$ & 14.7 & 4.4 & 16.3 & 2.1 & 1.4 & 3.5 \\
\hline $100(06: 40)$ & 14.7 & 4.3 & 16.5 & 2.1 & 1.6 & 3.4 \\
\hline $110(07: 20)$ & 14.2 & 4.2 & 16.9 & 2.0 & 1.7 & 3.6 \\
\hline $120(08: 00)$ & 14.5 & 4.0 & 16.7 & 1.6 & 1.8 & 3.9 \\
\hline $130(08: 40)$ & 14.4 & 3.7 & 16.8 & 1.6 & 1.6 & 3.1 \\
\hline $140(09: 20)$ & 14.1 & 3.7 & 16.8 & 1.6 & 2.0 & 3.1 \\
\hline $150(10: 00)$ & 14.2 & 3.8 & 17.0 & 2.0 & 2.5 & 2.4 \\
\hline $160(10: 40)$ & 12.8 & 3.7 & 16.6 & 2.3 & 2.1 & 2.3 \\
\hline $170(11: 20)$ & 12.9 & 3.4 & 16.0 & 2.4 & 2.5 & 1.6 \\
\hline $180(12: 00)$ & 12.5 & 2.6 & 15.9 & 2.2 & 3.4 & 2.0 \\
\hline $190(12: 40)$ & 12.0 & 3.0 & 15.3 & 2.8 & 2.7 & 2.0 \\
\hline $200(13: 20)$ & 13.0 & 3.0 & 15.8 & 2.9 & 2.9 & 1.4 \\
\hline $210(14: 00)$ & 12.8 & 3.2 & 16.1 & 3.0 & 2.6 & 2.1 \\
\hline $220(14: 40)$ & 12.5 & 3.7 & 16.3 & 3.4 & 2.4 & 1.9 \\
\hline $230(15: 20)$ & 12.6 & 3.7 & 15.4 & 3.2 & 2.4 & 1.2 \\
\hline $240(16: 00)$ & 14.0 & 3.4 & 15.5 & 3.3 & 1.4 & 0.6 \\
\hline $250(16: 40)$ & 15.3 & 2.0 & 16.8 & 1.8 & 1.5 & 1.3 \\
\hline $260(17: 20)$ & 15.0 & 2.2 & 17.4 & 1.7 & 1.5 & 1.2 \\
\hline $270(18: 00)$ & 15.4 & 1.6 & 17.0 & 1.3 & 1.0 & 0.8 \\
\hline $280(18: 40)$ & 16.3 & 2.6 & 17.5 & 2.3 & 1.7 & 1.1 \\
\hline Overall & 13.9 & 3.8 & 16.3 & 2.4 & 1.9 & 2.7 \\
\hline
\end{tabular}

The above results on boundary location are summarised in Table 2 where we list the median and standard deviation of the distribution of boundary positions at each longitude considered, and also in Fig. 5, which shows the median boundary positions plotted on a polar grid. Figure 5 has the same orientation as Fig. 1, i.e. the sunward direction is at the bottom of the figure and dawn to the left. Rings of co-latitude are marked at $5^{\circ}$ intervals and the red and blue crosses mark the median poleward and equatorward boundary positions, respectively, at every $10^{\circ}$ longitude for the range of longitudes considered in this study. It can be seen from this plot and the details in Table 2 that the average dawn side auroral oval is centred on $\sim 15^{\circ}-16^{\circ}$ co-latitude and has a width of $\sim 1^{\circ}-2^{\circ}$ at dawn. The average oval lies further poleward at noon, centred on $\sim 14^{\circ}$ co-latitude, and has a larger width of $\sim 3^{\circ}-4^{\circ}$. This widening is due to the occurrence of the "cusp-related" auroral features, as shown in Fig. 1d. Beyond $240^{\circ}$ longitude the equatorward and poleward boundary positions are both displaced $\sim 1^{\circ}$ equatorward relative to their positions at dawn, while overall the features are of similar width $\left(\sim 1^{\circ}-\right.$ $2^{\circ}$ at dusk). Over all longitudes considered in this study, the median poleward and equatorward locations are $13.9^{\circ}$ and $16.3^{\circ}$ co-latitude, with standard deviations of $3.8^{\circ}$ and $2.4^{\circ}$, respectively.

To discuss the width of the auroral oval in more detail, the widths calculated from the poleward and equatorward boundaries, "corrected" for the instrument PSF as described in Sect. 2.2, are plotted in Fig. 6 in a histogram format similar to Fig. 4. The median width values indicated by the vertical dashed lines show modest variation over longitude, from $\sim 3^{\circ}$ at $50^{\circ}-60^{\circ}$ longitude, reducing to $\sim 1^{\circ}-2^{\circ}$ in the range $80^{\circ}-140^{\circ}$ longitude i.e. at dawn and post-dawn. The median width becomes larger around noon, with a maximum of $3.4^{\circ}$ co-latitude at $180^{\circ}$ longitude, and then decreases to less than $2^{\circ}$ beyond $230^{\circ}$ longitude (15:20 LT) and remains around $\sim 1^{\circ}-2^{\circ}$ for the rest of the dusk region. The minimum median width observed is $1^{\circ}$ at $270^{\circ}$ longitude (dusk). The median width averaged over all longitudes is $1.9^{\circ}$ colatitude. Considering now the distributions of the auroral widths it can be seen that the width is very variable, covering a range of $<1^{\circ}-16^{\circ}$ co-latitude over all longitudes. This maximum range is observed in full in the post-dawn region at $110^{\circ}-120^{\circ}$ longitude, where the standard deviation is $\sim 4^{\circ}$, as listed in Table 2. Moving towards noon, the distribution 
narrows to $1^{\circ}-7^{\circ}$ (standard deviation $1.6^{\circ}$ ) at $170^{\circ}$ longitude, then broadens again post-noon, reaching a spread of $<1^{\circ}$ $9^{\circ}$ co-latitude (standard deviation $2.1^{\circ}$ ) at $210^{\circ}$ longitude. Beyond this in the dusk sector, the distribution is narrower i.e. $<1^{\circ}-5^{\circ}$ co-latitude, with a standard deviation of $\sim 1^{\circ}-$ $2^{\circ}$. The standard deviation averaged over all longitudes is $2.7^{\circ}$. The narrowest widths obtained on the dawn side correspond to discrete arcs of emission, similar to the one shown in Fig. 1b. The very wide auroral features $\left(13^{\circ}-16^{\circ}\right.$ wide $)$ measured on the dawn side are observed in the image on 26 January 2004 shown in Fig. 1d. No features this wide are observed on the dusk side, with a maximum width in this sector of $\sim 9^{\circ}$ at $210^{\circ}$ longitude (14:00 LT), due to a broad diffuse feature observed on 8 January 2004.

\section{Summary and discussion}

We have analysed a sample of 22 HST images of Saturn's southern auroral oval, observed during 1997-2004, to statistically determine the average location and width of the aurora, and their variability. Our results indicate that the median auroral oval in the pre-dawn to post-dusk region of Saturn's southern hemisphere, via noon, is centred on $\sim 15^{\circ}$ colatitude, with some modest variation over local time. The oval moves $\sim 1^{\circ}-2^{\circ}$ poleward around noon and is centred $\sim 1^{\circ}$ further equatorward at dusk relative to dawn. The median poleward boundary position is $\sim 14^{\circ}$ co-latitude over the range of longitudes considered, and the corresponding median equatorward boundary position is $\sim 16^{\circ}$ co-latitude. Values in the northern hemisphere are expected to be $\sim 1.5^{\circ}$ closer to the pole than these due to the interhemispheric asymmetry in the quadrupole term of the planet's internal magnetic field. The median width of the auroral oval is $\sim 2^{\circ}$ co-latitude, varying over local time from its narrowest of $\sim 1^{\circ}-2^{\circ}$ at dawn and dusk where discrete arcs of emission are observed (e.g. Fig. 1c), to the widest value of $\sim 3.5^{\circ}$ at noon, due to the presence of cusp-related diffuse emissions (e.g. Fig. 1d).

This study has also confirmed and quantified the variability in the location and width of Saturn's auroral oval. The poleward boundary of the aurora can be found between $2^{\circ}$ and $20^{\circ}$ co-latitude, with a standard deviation in position of $\sim 4^{\circ}$ co-latitude. The most poleward of these boundary locations correspond to a very bright diffuse feature observed on 26 January 2004 (Fig. 1e), which is also the broadest feature observed $\left(16^{\circ}\right.$ co-latitude wide), while the boundaries furthest from the pole occur when diffuse blobs of emission or narrow arcs are present (e.g. Figs. 1a and c). The equatorward boundary is correspondingly located between $6^{\circ}$ and $23^{\circ}$ co-latitude, with a standard deviation of $\sim 2.5^{\circ}$.

We now consider how these results compare with expectations based on the two principal auroral scenarios outlined in the Introduction, i.e. that the auroras are formed near the open-closed field line boundary with contributions
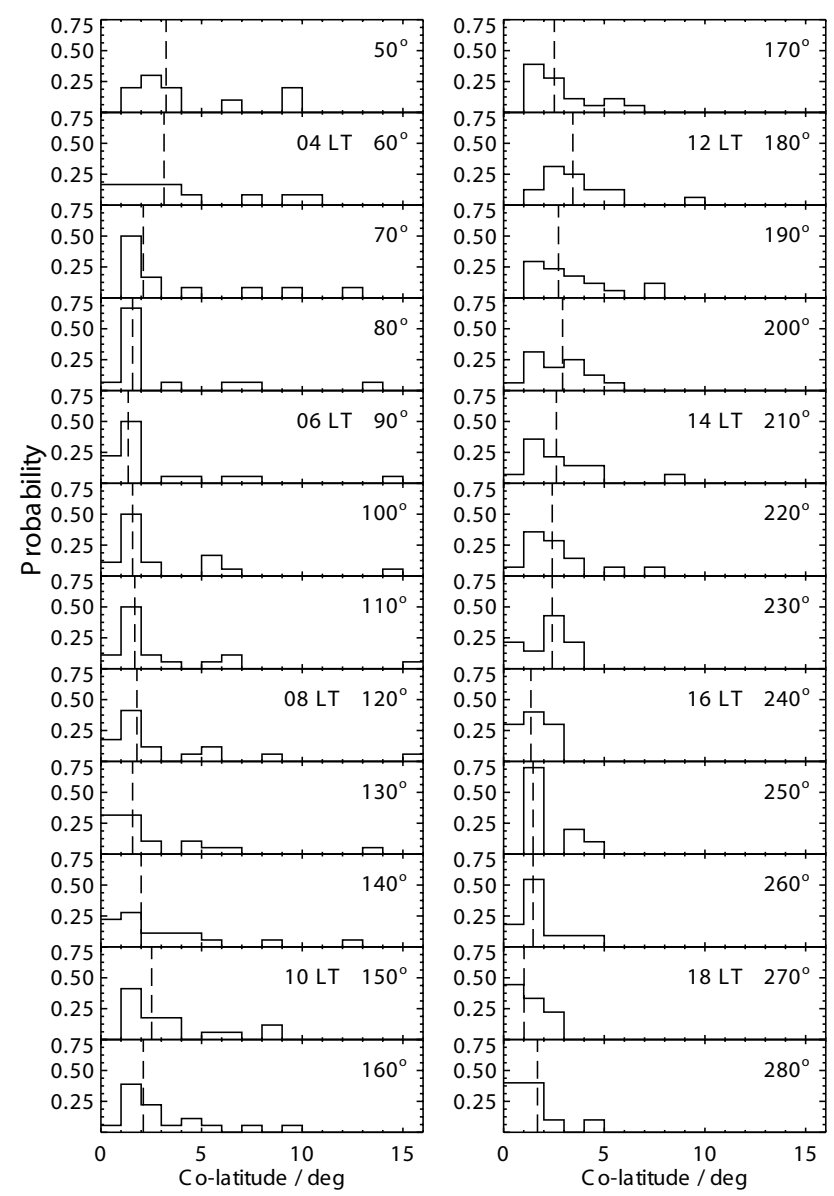

Fig. 6. Histograms showing the distribution of auroral oval latitudinal widths, plotted in a format similar to Fig. 4 . The vertical dashed lines mark the median width at each longitude.

potentially from both Dungey- and Vasyliunas-cycle dynamics, or that they may be associated with the upwarddirected field-aligned current system related to corotation breakdown deeper within the magnetosphere. To aid this discussion, Fig. 7 compares the co-latitudinal distribution of auroral boundaries derived here (lower panel) with magnetospheric plasma angular velocity data derived from Voyager observations by Richardson (1986) and Richardson and Sittler (1990) (upper panel), which have been normalised to the planet's angular velocity $\Omega_{S}$, and mapped into Saturn's southern ionosphere along model magnetic field lines. We note that related angular velocity data from the Cassini orbiter mission have yet to be published. These plasma data were obtained from the near-equatorial inbound passes of both Voyager-1 (green dots) and Voyager-2 (orange dots), both of which took place at post-noon local times between $\sim 13$ and $\sim$ 15:00 LT. The Voyager- 2 data span the radial range between the magnetopause at $\sim 19 R_{S}$ and $\sim 4 R_{S}$ in the inner magnetosphere, while the Voyager-1 data omit values obtained just inside the magnetopause at $\sim 23 R_{S}$ due 


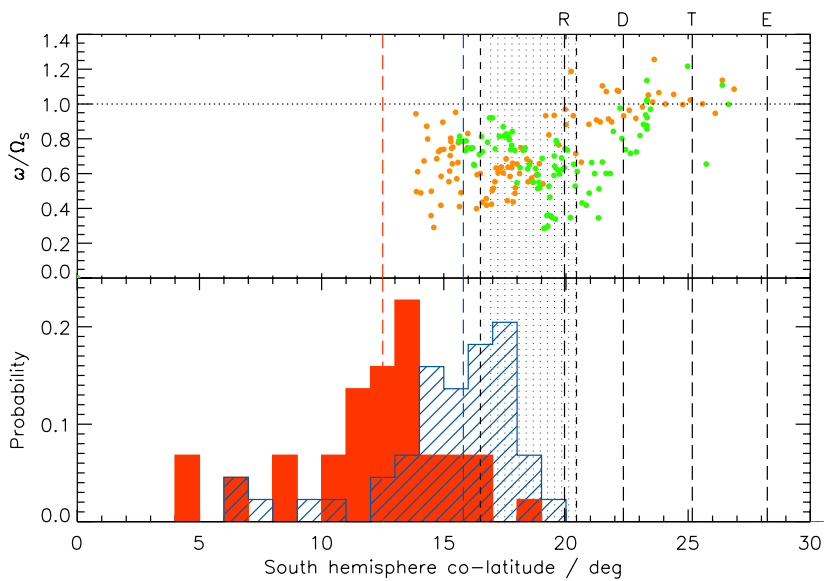

Fig. 7. Comparison of auroral boundary locations in the post-noon sector (lower panel), with Voyager plasma angular velocity data mapped magnetically into the southern ionosphere (upper panel). The format of the auroral data follows that of Fig. 4, but here the data from longitudes $200^{\circ}, 210^{\circ}$, and $220^{\circ}$ (13:20-14:40 LT) have been added together to match the local time range of the Voyager data. The data in the upper panel were obtained from the inbound passes of Voyager-1 (green dots) and Voyager-2 (orange dots), both spanning the local time range $\sim 13: 00$ to $\sim 15: 00$ LT. The angular velocities have been normalised to the planetary angular velocity $\Omega_{S}$, with rigid corotation, $\left(\omega / \Omega_{S}\right)=1$, being indicated by the horizontal dotted line. The values have been mapped into the southern ionosphere using a magnetic model consisting of the SPV internal field model and the Connerney et al. (1983) Voyager ring current model. The mapped range of the ring current is shown stippled, lying between 8 and 15.5 $R_{S}$ in the equatorial plane, while the vertical black dashed lines show the mapped orbits of the icy moons Rhea (R), Dione (D), Tethys (T), and Enceladus (E).

to uncertainty in the identity of the ion species observed in this region, and cover the radial range between $\sim 17 R_{S}$ and $\sim 5 R_{S}$. The magnetic model employed to map these data to the ionosphere consists of the sum of the SPV internal field model derived by Davis and Smith (1990), and the Connerney et al. (1983) model of Saturn's ring current fitted specifically to the Voyager magnetic field data. In this model, the ring current region extends in the equatorial plane between 8 and $15.5 R_{S}$, the corresponding extent in the ionosphere being shown in Fig. 7 by the stippled region bounded by the black dashed lines. The combined magnetic model provides a good description of the magnetic data out to distances a few $R_{S}$ outside the ring current region, mapping in the southern ionosphere to $\sim 15^{\circ}$ co-latitude, but because magnetopause currents are not included in the model, the mapping becomes more uncertain at distances beyond (i.e. at colatitudes smaller than $\sim 15^{\circ}$ ). To provide further context, the black vertical dashed lines also show the mapped positions of the orbits of the larger icy moons that orbit within the equatorial magnetosphere, Enceladus (E) at $3.9 R_{S}$, Tethys (T) at $4.9 R_{S}$, Dione (D) at $6.3 R_{S}$, and Rhea (R) at $8.7 R_{S}$, where we note the recent evidence from Cassini that Enceladus is most probably the principal source of (water) gas and plasma in Saturn's magnetosphere (Hansen et al., 2006; Waite et al., 2006). We also note that mapping "to the ionosphere" here specifically means to the surface $1000 \mathrm{~km}$ above the planetary 1 bar reference spheroid, as has also been employed in the mapping of the auroral data.

The histograms of auroral boundary locations shown in the bottom panel of Fig. 7 follow the same format as Fig. 4, but combine data for longitudes between $200^{\circ}$ and $220^{\circ}$ (local times between 13:20 and 14:40 LT) in order to match the local time of the Voyager data. The vertical red and blue dashed lines indicate the median positions of the boundaries as before, showing that the auroral oval at these local times lies typically at (southern) co-latitudes between $\sim 12.5^{\circ}$ and $\sim 15.8^{\circ}$ in agreement with Fig. 5. The median equatorward boundary maps magnetically to the equatorial plane at radial distances of $\sim 16.5 R_{S}$, just outside the stippled Connerney et al. (1983) ring current region, according to our magnetic model. The median poleward auroral boundary then lies poleward of the data from Voyager-2, the most poleward of which maps to the vicinity of the magnetopause as indicated above, and hence to the region just equatorward of the boundary between open and closed field lines. The region sufficiently to the left of the Voyager data then represents open field lines, unexplored on the near-equatorial inbound spacecraft trajectories. While noting the relative uncertainty in the mapping of the outer Voyager- 2 data points mentioned above, the results in Fig. 7 indicate that it is at least plausible that the main auroral oval at these local times maps typically into the outermost layer of closed magnetospheric field lines beyond the ring current, and possibly onto open field lines as well, associated for example with emissions from the dayside cusp. This inference is also compatible with the estimates of Ness et al. (1981) based on Voyager magnetotail data, that the open-closed field line boundary at Saturn lies in the range $\sim 11^{\circ}-15^{\circ}$ co-latitude, this coinciding with the central part of the distribution of poleward boundary positions in Fig. 7.

Looking further at the angular velocity data in the upper panel of Fig. 7, it can be seen that the magnetospheric plasma significantly sub-corotates within the (stippled) ring current region at $\sim 50-80 \%$ of rigid corotation. The plasma angular velocities only begin to rise toward rigid corotation (the horizontal dotted line) near the inner edge of the ring current and at smaller equatorial radial distances. Specifically, the Voyager-2 data start to rise toward rigid corotation near the orbit of Rhea lying just inside the inner ring current region at $\sim 9 R_{S}$ in the equatorial plane, mapping to $\sim 20^{\circ}$ co-latitude in the ionosphere, and appear to be consistently near rigid corotation inside $\sim 6 R_{S}$ near Dione's orbit, mapping to $\sim 23^{\circ}$ co-latitude. The Voyager-1 data indicate significant subcorotation to even smaller equatorial distances of $\sim 6.5 R_{S}$, mapping to $\sim 22^{\circ}$ co-latitude, only rising toward rigid corotation inside $\sim 5.5 R_{S}$, mapping to $\sim 24^{\circ}$. If we then consider the consequences for field-aligned current flow associated 
with variations in the equatorward-directed Pedersen current driven in the ionosphere by plasma sub-corotation, it can firstly be seen that there is much scope for small-scale structures to be present throughout the sub-corotating region associated with small-scale variations in the plasma angular velocity, as discussed previously and modelled by Cowley and Bunce (2003c) and Cowley et al. (2004b). However, the main upward-directed field-aligned current, equivalent to that believed to be associated with the main oval at Jupiter, will flow where the plasma angular velocity increases from significant sub-corotation in the ring current region (and beyond) towards consistent near-rigid corotation at smaller radial distances. The Voyager- 1 and -2 data in Fig. 7 then indicate that this occurs between equatorial radial distances of $\sim 9$ and $\sim 6 R_{S}$, mapping to $\sim 20^{\circ}-24^{\circ}$ co-latitude in the ionosphere. These locations lie well equatorward of the typical position of the auroras indicated by the median boundaries, indeed, they do not overlap the auroral distribution at these local times at all. It thus seems reasonable to conclude on this basis that Saturn's auroral oval is most probably located near the open-closed field line boundary, rather than being related to the main corotation-enforcement current system as is believed to be the case at Jupiter, in agreement with the earlier conclusions of Cowley and Bunce (2003c) and Cowley et al. (2004b). In this case we expect the auroras at Saturn to be associated with Dungey-cycle and possibly Vasyliunas-cycle dynamics, thus also explaining the persistent dawn-dusk local time asymmetries which are observed, as can be seen in Fig. 1 (Cowley et al., 2004a, 2005; Jackman and Cowley, 2006).

The other major feature of our results concerns the considerable variability in the position of the auroral boundaries at Saturn, contrasting sharply with the behaviour of Jupiter's main oval, which varies in latitude at a given planetary longitude by at most $\sim 2^{\circ}$ even during intervals of strong solar wind disturbance (Grodent et al., 2003; Nichols et al., 2006). However, if the auroras at Saturn are located near the openclosed field line boundary, as discussed above, this variability is reasonably explained in terms of variations in the amount of open flux present in the system. An indication of the magnitude of the changes in open flux which may be anticipated to occur in the Dungey-cycle at Saturn can be obtained by considering the corresponding variations at Earth. In a study of a very active interval Milan et al. (2004) found that the open flux in the terrestrial system varied between $\sim 2.5 \%$ and $\sim 12 \%$ of the total planetary magnetic flux, where the minimum value followed a strong magnetospheric compression by the solar wind and the onset of a large substorm. Subsequent analysis of a larger data set by Milan et al. (2006) has confirmed that the above values fully span the usual range of open flux fractions at Earth. The corresponding range at Saturn cannot be inferred in a simple way from the range in poleward boundary locations found above in our study, because the highest-latitude boundaries in particular are associated with asymmetric auroral distributions occurring during disturbed intervals, as can be seen in Fig. 1. However, Badman et al. (2005) numerically computed the magnetic flux present in the "dark" region bounded by the auroral oval at Saturn using the data set obtained during the HST-Cassini campaign in January 2004, which spanned nearly a full solar rotation. They found that the flux varied between $\sim 13$ and $\sim 49 \mathrm{GWb}$, corresponding to between $\sim 3 \%$ and $\sim 10 \%$ of the total flux threading the ionosphere $(\sim 475 \mathrm{GWb})$. The smallest values again correspond to disturbed conditions following the impact of a solar wind corotating interaction region compression on the magnetosphere. The close correspondence between these fractional values and those observed at Earth at least lends plausibility to the open flux interpretation of the "dark" polar region at Saturn.

If we accept the conclusion of the above discussion, then the poleward boundary of the auroral emission at Saturn can be used as a proxy for the boundary between open and closed field lines, as assumed in the earlier study of Badman et al. (2005). On the dayside this boundary thus maps magnetically to the vicinity of the dayside magnetopause. Very narrow arcs at this boundary, such as that shown in Fig. 1c, may then correspond simply to a region of upwarddirected field-aligned current, as, for example, in the theoretical model of Jackman and Cowley (2006). However, it seems reasonable to suppose that broader features, such as those seen e.g. in Figs. 1a, b, and f, can also be formed by precipitation from regions of hot magnetospheric plasma on closed flux tubes, that may be injected on the nightside and then sub-corotate around the boundary into the dayside magnetosphere. In this case, the width of the auroral features reflects the radial extent of the hot plasma region adjacent to the magnetopause boundary, which can then be estimated from the oval width by conservation of magnetic flux. We consider a segment of the auroral oval of azimuthal extent $\Delta \varphi$, located at a co-latitude $\theta$, and of latitudinal width $\Delta \theta$. The magnetic flux threading this region of the ionosphere is then $\Delta \Phi=B_{i} R_{i}^{2} \sin \theta \Delta \theta \Delta \varphi$, where $B_{i}$ is the magnitude of the near-radial magnetic field in the ionosphere, and $R_{i}$ is the ionospheric radius. Assuming that this maps magnetically into the equatorial plane in a region of width $l$ adjacent to the magnetopause at radius $R_{M P}$, then we also have $\Delta \Phi \approx B_{e} l R_{M P} \Delta \varphi$, where $B_{e}$ is the equatorial field strength, and we have assumed for simplicity that the field lines lie in meridian planes. Equating magnetic fluxes we then find

$l \approx \frac{B_{i}}{B_{e}} \frac{R_{i}^{2}}{R_{M P}} \sin \theta \Delta \theta$.

The radius of the polar ionospheric layer is $R_{i} \approx 55400 \mathrm{~km}$ ( $\sim 1000 \mathrm{~km}$ above the 1-bar reference spheroid), while the radial field strength in the southern ionosphere is $B_{i} \approx 60000 \mathrm{nT}$ according to the SPV model (Davis and Smith, 1990). We also take typical equatorial values of $R_{M P} \approx 22 R_{S}$ and $B_{e} \approx 5 \mathrm{nT}$. Then for a typical auroral distribution with $\theta \approx 15^{\circ}$ and $\Delta \theta \approx 2^{\circ}$ (the median values found in our study), we find a value $l \approx 4 R_{S}$. This value may be 
somewhat reduced if the dayside field lines spread meridionally away from noon as they do at Earth (e.g. Fairfield, 1968). Nevertheless, we may conclude that the auroral widths found here imply the existence of hot plasma layers inside the magnetopause whose width is typically several planetary radii, which occupy some fraction of the region between the magnetopause and the outer edge of the ring current, as in the above discussion. It will be interesting in future work to test this inference using in situ plasma data from the Cassini spacecraft.

Acknowledgements. This work is based on observations made by the NASA/ESA Hubble Space Telescope, obtained at the Space Telescope Science Institute, which is operated by the AURA Inc for NASA. We thank J. T. Clarke for use of the January 2004 HST images. The Voyager data shown in Fig. 7 were supplied by the Planetary Data Center at UCLA. Research at Leicester was supported by PPARC grant PPA/G/O/2003/00013. S. V. Badman was supported by a PPARC studentship and S. W. H. Cowley by a Royal Society Leverhulme Trust Senior Research Fellowship. J.-C. Gérard and D. Grodent were supported by the Belgian Fund for Scientific Research (FNRS) and the PRODEX programme of the European Space Agency.

Topical Editor I. A. Daglis thanks D. G. Mitchell and another referee for their help in evaluating this paper.

\section{References}

Badman, S. V., Bunce, E. J., Clarke, J. T., Cowley, S. W. H., Gérard, J.-C., Grodent, D., and Milan, S. E.: Open flux estimates in Saturn's magnetosphere during the January 2004 Cassini-HST campaign, and implications for reconnection rates, J. Geophys. Res., 110, A11216, doi:10.1029/2005JA011240, 2005.

Broadfoot, A. L., Sandel, B. R., Shemansky, D. E., Holberg, J. B., Smith, G. R., Strobel, D. F., McConnell, J. C., Kumar, S., Hunten, D. M., Atreya, S. K., Donahue, T. M., Moos, H. W., Bertaux, J. L., Blamont, J. E., Pomphrey, R. B., and Linik, S.: Extreme ultraviolet observations from Voyager 1 encounter with Saturn, Science, 212, 206-211, 1981.

Bunce, E. J., Cowley, S. W. H., and Milan, S. E.: Interplanetary magnetic field control of Saturn's polar cusp aurora, Ann. Geophys., 23, 1405-1431, 2005, http://www.ann-geophys.net/23/1405/2005/.

Clarke, J. T., Moos, H. W., Atreya, S. K., and Lane, A. L.: IUE detection of bursts of H Ly $\alpha$ emission from Saturn, Nature, 290, 226-230, 1981.

Connerney, J. E. P., Acuña, M. H., and Ness, N. F.: Currents in Saturn's magnetosphere, J. Geophys. Res., 88, 8779-8789, 1983.

Cowley, S. W. H. and Bunce, E. J.: Origin of the main auroral oval in Jupiter's coupled magnetosphere-ionosphere system, Planet. Space Sci., 49, 1067-1088, 2001.

Cowley, S. W. H. and Bunce, E. J.: Modulation of jovian middle magnetosphere currents and auroral precipitation by solar windinduced compressions and expansions of the magnetosphere: Initial conditions and steady state, Planet. Space Sci., 51, 31-56, 2003a.

Cowley, S. W. H. and Bunce, E. J.: Modulation of Jupiter's main auroral oval emissions by solar wind-induced expansions and com- pressions of the magnetosphere, Planet. Space Sci., 51, 57-79, 2003b.

Cowley, S. W. H. and Bunce, E. J.: Corotation-driven magnetosphere-ionosphere coupling currents in Saturn's magnetosphere and their relation to the auroras, Ann. Geophys., 21, 1691-1707, 2003c.

Cowley, S. W. H., Bunce, E. J., and Prangé, R.: Saturn's polar ionospheric flows and their relation to the main auroral oval, Ann. Geophys., 22, 1379-1394, 2004a.

Cowley, S. W. H., Bunce, E. J., and O'Rourke, J. M.: A simple quantitative model of plasma flows and currents in Saturn's polar ionosphere, J. Geophys. Res., 109, A05212, doi:10.1029/2003JA010375, 2004b.

Cowley, S. W. H., Badman, S. V., Bunce, E. J., Clarke, J. T., Gérard, J.-C., Grodent, D., Jackman, C. M., Milan, S. E., and Yeoman, T. K.: Reconnection in a rotation-dominated magnetosphere and its relation to Saturn's auroral dynamics, J. Geophys. Res., 110, A02201, doi:10.1029/2004JA010796, 2005.

Davis Jr., L. and Smith, E. J.: A model of Saturn's magnetic field based on all available data, J. Geophys. Res., 95, 15 257-15 261, 1990.

Dungey, J. W.: Interplanetary field and the auroral zones, Phys. Rev. Lett., 6, 47-48, 1961.

Fairfield, D. H.: Average magnetic field configuration of the outer magnetosphere, J. Geophys. Res., 73, 7329-7338, 1968.

Gérard, J.-C., Dols, V., Grodent, D., Waite, J. H., Gladstone, G. R., and Prangé, R.: Simultaneous observations of the saturnian aurora and polar haze with the HST/FOC, Geophys. Res. Lett., 22, 2685-2688, 1995.

Gérard, J.-C., Grodent, D., Gustin, J., Saglam, A., Clarke, J. T., and Trauger, J. T.: Characteristics of Saturn's FUV aurora observed with the Space Telescope Imaging Spectrograph, J. Geophys. Res., 109, A09207, doi:10.1029/2004JA010513, 2004.

Gérard, J.-C., Bunce, E. J., Grodent, D., Cowley, S. W. H., Clarke, J. T., and Badman, S. V.: Signature of Saturn's auroral cusp: Simultaneous HST FUV observations and upstream solar wind monitoring, J. Geophys. Res., 110, A11201, doi:10.1029/2005JA011094, 2005.

Gérard, J.-C., Grodent, D., Cowley, S. W. H., Mitchell, D. G., Kurth, W. S., Clarke, J. T., Bunce, E. J., Nichols, J. D., Dougherty, M. K., Crary, F. J., and Coates, A. J.: Saturn's auroral morphology and activity during quiet magnetospheric conditions, J. Geophys. Res., in press, doi:10.1029/2006JA011965, 2006.

Gong, B. and Hill, T. W.: Variations of jovian and saturnian auroras induced by changes of solar wind dynamic pressure, Magnetospheres of the Outer Planets 2005 Programme and Abstracts, p. 129, University of Leicester, Leicester, UK, 2005.

Grodent, D., Clarke, J. T., Kim, J., Waite Jr., J. H., and Cowley, S. W. H.: Jupiter's main auroral oval observed with HST-STIS, J. Geophys. Res., 108(A11), 1389, doi:10.1029/2003JA009921, 2003.

Grodent, D., Gérard, J.-C., Cowley, S. W. H., Bunce, E. J., and Clarke, J. T.: The global morphology of Saturn's southern ultraviolet aurora, J. Geophys. Res., 110, A07215, doi:10.1029/2004JA010983, 2005.

Hansen, C. J., Esposito, L., Stewart, I. A. F., Colwell, J., Hendrix, A., Pryor, W., Shemansky, D., and West, R.: Enceladus' water vapor plume, Science, 311, 422-425, 2006. 
Hill, T. W.: Inertial limit on corotation, J. Geophys. Res., 84, 65546558, 1979.

Hill, T. W.: The jovian auroral oval, J. Geophys. Res., 106, 81018108, 2001.

Jackman, C. M. and Cowley, S. W. H.: A model of the plasma flow and current in Saturn's polar ionosphere under conditions of strong Dungey-cycle driving, Ann. Geophys., 24, 1029-1055, 2006 , http://www.ann-geophys.net/24/1029/2006/.

Judge, D. L., Wu, F. M., and Carlson, R. W.: Ultraviolet photometer observations of the Saturnian system, Science, 207, 431-434, 1980.

McGrath, M. A. and Clarke, J. T.: H I Lyman alpha emission from Saturn (1980-1990), J. Geophys. Res., 97, 13 691-13 703, 1992.

Milan, S. E., Lester, M., Cowley, S. W. H., Oksavik, K., Brittnacher, M., Greenwald, R. A., Sofko, G., and Villain, J.-P.: Variations in polar cap area during two substorm cycles, Ann. Geophys., 21, 1121-1140, 2003, http://www.ann-geophys.net/21/1121/2003/.

Milan, S. E., Cowley, S. W. H., Lester, M., Wright, D. M., Slavin, J. A., Fillingim, M., Carlson, C. W., and Singer, H. J.: Response of the magnetotail to changes in the open flux content of the magnetosphere, J. Geophys. Res., 109, A04220, doi:10.1029/2003JA010350, 2004.

Milan, S. E., Provan, G., and Hubert, B.: Magnetic flux transport in the Dungey-cycle: A survey of dayside and nightside reconnection rates, J. Geophys. Res., in press, doi:10.1029/2006JA011642, 2006.

Ness, N. F., Acuña, M. H., Lepping, R. P., Connerney, J. E. P., Behannon, K. W., Burlaga, L. F., and Neubauer, F. M.: Magnetic field studies by Voyager 1: Preliminary results at Saturn, Science, 212, 211-217, 1981.

Nichols, J. D., Bunce, E. J., Clarke, J. T., Cowley, S. W. H., Gérard, J.-C., Grodent, D., and Pryor, W. R.: Response of Jupiter's UV auroras to interplanetary conditions as observed by the Hubble Space Telescope during the Cassini fly-by campaign, J. Geophys. Res., in press, doi:10.1029/2006JA012005, 2006.
Prangé, R., Pallier, L., Hansen, K. C., Howard, R., Vourlidas, A., Courtin, R., and Parkinson, C.: An interplanetary shock traced by planetary auroral storms from the Sun to Saturn, Nature, 432, 78-81, 2004.

Richardson, J. D.: Thermal ions at Saturn: plasma parameters and implications, J. Geophys. Res., 91, 1381-1389, 1986.

Richardson, J. D. and Sittler Jr., E. C.: A plasma density model for Saturn based on Voyager observations, J. Geophys. Res., 95, 12 019-12 031, 1990.

Sandel, B. R. and Broadfoot, A. L.: Morphology of Saturn's aurora, Nature, 292, 679-682, 1981.

Sandel, B. R., Shemansky, D. E., Broadfoot, A. L., Holberg, J. B., Smith, G. R., McConnell, J. C., Strobel, D. F., Atreya, S. K., Donahue, T. M., Moos, H. W., Hunten, D. M., Pomphrey, R. B., and Linik, S.: Extreme ultraviolet observations from the Voyager 2 encounter with Saturn, Science, 215, 548-553, 1982.

Southwood, D. J. and Kivelson, M. G.: A new perspective concerning the influence of the solar wind on Jupiter, J. Geophys. Res. 106, 6123-6130, 2001.

Trauger, J. T., Clarke, J. T., Ballester, G. E., Evans, R. W., Burrows, C. J., Crisp, D., Gallagher III, J. S., Griffiths, R. E., Hester, J. J., Hoessel, J. G., Holtzman, J. A., Krist, J. E., Mould, J. R., Sahai, R., Scowen, P. A., Stapelfeldt, K. R., and Watson, A. M.: Saturn's hydrogen aurora: Wide field and planetary camera 2 imaging from the Hubble Space Telescope, J. Geophys. Res., 103, 20 237-20 244, 1998.

Vasyliunas, V. M.: Plasma distribution and flow, in: Physics of the Jovian Magnetosphere, edited by: Dessler, A. J., p. 395-453, Cambridge Univ. Press, Cambridge, UK, 1983.

Waite Jr., J. H., Combi, M. R., Ip, W.-H., Cravens, T. E., McNutt Jr, R. L., Kasprzak, W., Yelle, R., Luhmann, J., Niemann, H., Gell, D., Magee, B., Fletcher, G., Lunine, J., and Tseng, W.-L.: Cassini Ion and Neutral Mass Spectrometer: Enceladus plume composition and structure, Science, 311, 14191422, 2006. 